\title{
ON THE RESOLVING POWER OF A PRISM SPECTROMETER FOR THE INFRARED
}

\author{
By John Strong* \\ California Institute of Technology, Pasadena \\ (Received January 15, 1931)
}

Abstract

The resolving power of a spectrometer in the infrared is limited by the rate at which energy passes through the exit slit of the spectrometer. Calculation of the energy available for activating a thermocouple, under condition that the exit slit embrace the same frequency range $\Delta \nu$ of spectrum at all times, gives information on the attainable resolving power at various wave-lengths and with various prisms. The best dispersing angle for a sylvine prism to be used from 8 to $22 \mu$ is from $70^{\circ}$ to $75^{\circ}$. A lamellar prism construction is described.

\section{INTRODUCTION}

IN THE spectral region where a photographic plate, which measures the energy received during the time of exposure, is used, the resolving power of a spectrometer is limited by interference effects. However, in the infrared where a thermocouple or radiometer is used one does not measure the energy received over an arbitrary period of time but the rate at which energy is received. This available energy rather than interference effects places the limit on the resolving power of the spectrometer.

It was the purpose of this investigation to make calculations of the energy available for activating a receiver (such as a thermocouple). The energy of a continuous spectrum passing through the exit slit, with both slits equal, is proportional to the square of the slit width, so that if the value for the available energy at any particular wave-length and for a particular dispersing prism is greater, by a factor $\alpha$, than the energy necessary to give a definite response of the receiving device, then the spectrometer slits may be closed by a factor $1 / \alpha^{1 / 2}$ and still give a practical deflection. The available energy is therefore a measure of the resolving power which is attainable under varying conditions as long as the limit set by interference effects is not exceeded.

The calculation of available energy is instructive on account of the information that it gives regarding the resolving power attainable in various regions of the spectrum. The determination of the absorption spectrum of a gas in the infrared consists of a study of the heat radiations emitted from some convenient source and the changes in spectral energy distribution which result from the introduction of the gas into the radiation path. The spectrum emitted from a heated body possesses very little energy at long wave-lengths since the emission cannot exceed that from a black body which varies inversely with the fourth power of the wave-length. This scarcity of available

* National Research Fellow. 
energy at long wave-lengths would seem to place a serious limitation upon the character of experimental work that might be done at long wave-lengths. However, the results of Robertson and Fox ${ }^{1}$ with a rocksalt prism on the oscillation-rotation spectrum of ammonia and similar molecules shows a definite improvement in resolution at the longer wave-lengths. These calculations will show in a quantitative way that similar improvement at long wavelengths is to be expected for a sylvine prism.

The calculation of available energy for various prisms shows the best prism to use for a particular spectral region. For the entire spectral range for which sylvine is a useful prism material, it is best to have as the dispersing angle of the prism 70 to 75 degrees. Usually a $60^{\circ}$ prism is considered best but this is neither obvious nor a consequence of the classical works of Pickering $^{2}$ and of Wadsworth. ${ }^{3}$ These authors made calculations of the best dimensions for prisms made from materials having values for the refractive index varying between 1.5 and 1.8. Their calculations do not hold because sylvine has refractive indices below 1.5 in the infrared, also the formula used by Pickering and Wadsworth for the transmission of a prism is invalid except when the absorption is very low.

\section{Priliminary Considerations}

When a band system in the infrared is plotted with wave number rather than wave-length as the abscissa one gets a more natural picture, since the fineness of structure is then approximately the same throughout the spectrum. On the other hand, if wave-length is used as abscissa, the structure appears coarser at longer wave-lengths and in fact if $\Delta \nu$ is taken as a measure of the fine structure of the band system the corresponding $\Delta \lambda$ becomes greater at longer wave-lengths according to the formula

$$
\Delta \lambda=\frac{\lambda^{2} \Delta \nu}{c} .
$$

This means that if the slit of the spectrometer is adjusted so that the wavelength interval in the slit is increased proportionally with $\lambda^{2}$ it will embrace the same frequency range of spectrum. The energy passing through the exit slit is proportional to the spectral energy density of illumination of the slit, $E_{\lambda}$, multiplied by the square of the slit width. For a black body at long wavelengths $E_{\lambda}$ is proportional to $\lambda^{-4}$ while for an ideal spectrometer having constant dispersion and constant efficiency the slit width, $s$, would be proportional to $\lambda^{2}$ and $E_{\lambda} s^{2}$, the energy passing through the exit slit, would be independent of $\lambda$. By constant efficiency it is meant that the same fraction of the energy of various wave-lengths is lost by reflection at the various surfaces in the optical path and by absorption in the prism. This means that the resolving power measured by the rational magnitude $\Delta \nu$ will be the same throughout the spectrum.

${ }^{1}$ R. Robertson and J. J. Fox, Proc. Roy. Soc. A120, 128 (1928).

${ }^{2}$ Pickering, Phil. Mag (4), 36, 39 (1868).

${ }^{3}$ F. L. O. Wadsworth, Astrophys. J. 2, 264 (1895) 
For a spectrometer which is not ideal the energy passing through the exit slit increases as the dispersion increases and decreases as the efficiency decreases. $\delta n / \delta \lambda$ for the materials transparent in the infrared increases at longer wave-lengths while the transparency decreases. The dispersion factor predominates at first and so the available energy (when the slit width is constant as measured by $\Delta \nu$ ) increases and greater resolving power is attainable at longer wave-lengths. This for sylvine is a result similar to the experimental results of Robertson and Fox. The available energy passes through a maximum and rapidly decreases as the transparency factor becomes predominate. When the dispersing angle of the prism increases the dispersion increases, at first slowly, then rapidly, while the light lost by reflection increases. It was for the purpose of arriving at the best compromise between these varying factors that the following calculations were made.

\section{Energy Available to Activate Receiver}

For a simple prism spectrometer the energy, $e$, passing through the exit slit is given by the following expression

$$
e=E_{\lambda} s_{1} l_{1} \frac{A}{f_{1}{ }^{2}} \frac{s_{2}}{f_{2} \frac{d \theta}{d \lambda}} T F R .
$$

The symbols are defined by the following formulas:

$E_{\lambda}$ is the spectral energy density of illumination of the entrance slit and for long wave-lengths this is given by the Rayleigh-Jeans formula.

$$
E_{\lambda}=\frac{4 \pi k t}{\lambda^{4}} .
$$

$s_{1}$ and $l_{1}$, are width and length respectively of the entrance slit.

$A$ is the effective area of the collimating mirror of the spectrometer. For a large collimating mirror this is the area of the prism face $(h \times b$ if $h$ is the heighth of prism face and $b$ the length) projected on the plane of the mirror face. If $i$ is the angle of incidence of the light on the prism face, $A$ is defined by the equation $A=h \times b \cos i$.

$f_{1}$ and $f_{2}$ are the focal lengths respectively of the collimating and telescope mirrors.

$s_{2}$ is the width of the exit slit.

$d \theta / d \lambda$ is the dispersion of the prism and it may be broken up into two factors $(\partial \theta / \delta n) \times \partial n / \partial \lambda$. For a prism at minimum deviation $\partial \theta / \partial n$ is given by $2 \sin (\phi / 2) / \cos i$ where $\phi$ is the dispersing angle of the prism. Values of $\partial n / \partial \lambda$ are defined by the empirical formula given by Rubens ${ }^{4}$ for the index of refraction of sylvine.

$T$ is the transmission of the prism. This is given by the formula

$$
T=\frac{1}{\kappa \tau}\left(1-e^{-\kappa \tau}\right)
$$

${ }^{4}$ Rubens, Ann. d. Physik 54, 476 (1895); 60, 724 (1897). 
The absorption coefficient is represented by $\kappa$ and the width of the prism opposite the dispersing angle is represented by $\tau$. For values of $\kappa \tau$ less than 0.1 the values of $T$ are given approximately by the formula used by Pickering and Wadsworth in their work, $T=e^{-\kappa \tau / 2}$. For values of $\kappa \tau$ greater than 5 the values of $T$ are given approximately by $1 / \kappa \tau$. Table I gives values of $T$ for various values of $\kappa \tau$.

TABLE I.

\begin{tabular}{rrrrrrrr}
\hline \hline$\kappa \tau$ & $T$ & $\kappa \tau$ & $T$ & $\kappa \tau$ & \multicolumn{1}{c}{$T$} & $\kappa \tau$ & \multicolumn{1}{c}{$T$} \\
\hline 0.01 & 0.995 & 0.11 & 0.946 & 0.1 & 0.952 & 1 & 0.632 \\
.02 & .989 & .12 & .943 & .2 & .906 & 2 & .432 \\
.03 & .985 & .13 & .938 & .3 & .864 & 3 & .317 \\
.04 & .980 & .14 & .934 & .4 & .824 & 4 & .245 \\
.05 & .975 & .15 & .929 & .5 & .787 & 5 & .198 \\
.06 & .970 & .16 & .924 & .6 & .752 & 6 & .166 \\
.07 & .966 & .17 & .919 & .7 & .719 & 7 & .143 \\
.08 & .961 & .18 & .915 & .8 & .688 & 8 & .125 \\
.09 & .956 & .19 & .911 & .9 & .659 & 9 & .111 \\
.10 & .952 & .20 & .906 & 1.0 & .632 & 10 & .100 \\
\hline \hline
\end{tabular}

$F$ is a factor which takes account of the loss of light due to reflections at the two prism surfaces and is represented by the following formula,

$$
F=\left\{\frac{1}{2}\left[1-\frac{\sin ^{2}(i-r)}{\sin ^{2}(i+r)}\right]+\frac{1}{2}\left[1-\frac{\tan ^{2}(i-r)}{\tan ^{2}(i+r)}\right]\right\}^{2}
$$

where $r$ is the angle of refraction at the prism face.

$R$ is the total reflectivity of all the spectrometer mirrors and is considered constant. For eight normal reflections from polished silver the loss of light is 8 percent at $10 \mu$ and 4 percent at $20 \mu$.

If $f_{1}=f_{2}$ and if the spectrometer slits are equal and always adjusted so that the frequency interval they subtend is constant then $s_{1}$ and $s_{2}$ may be substituted for by the expression

$$
s_{2}=s_{1}=f_{1} \frac{d \theta}{d \lambda} \frac{\lambda^{2} \Delta \nu_{0}}{c}
$$

where $\Delta \nu_{0}$ corresponds to the constant frequency interval embraced by the slits.

Substituting in the formula for $e$ gives

$$
e=\frac{8 \pi k t \Delta \nu_{0}^{2} h b R}{c^{2} f_{1}} T F \frac{\partial n}{\partial \lambda} \sin \frac{\phi}{2}
$$

or

$$
e \propto \epsilon=T F \frac{\partial n}{\partial \lambda} \sin \frac{\phi}{2} .
$$

Values of $F$ for various prism angles, $\phi$, and various wave-lengths are given in Table II. For the calculation of $\epsilon$ given in Table III, $\tau$ was given the value 10 centimeters. 
TABLE II. Values of F for various $\phi$ and $\lambda$.

\begin{tabular}{cccccc}
\hline \hline$\phi$ & $10.02 \mu$ & $14.14 \mu$ & $17.68 \mu$ & $20.6 \mu$ & $22.5 \mu$ \\
\hline $0^{\circ}$ & 0.931 & 0.936 & 0.942 & 0.947 & 0.971 \\
$60^{\circ}$ & .910 & .917 & .925 & .934 & .966 \\
$70^{\circ}$ & .873 & .885 & .898 & .901 & .957 \\
$75^{\circ}$ & .799 & .847 & .846 & .886 & .950 \\
$80^{\circ}$ & .727 & .768 & .805 & .840 & .937 \\
$85^{\circ}$ & .419 & .563 & .662 & .735 & .915 \\
$90^{\circ}$ & - & - & - & .244 & .877 \\
\hline \hline
\end{tabular}

TABLE III. Values of $\epsilon$ for various $\phi$ and $\lambda$.

\begin{tabular}{crrrrrrrrr}
\hline \hline$\phi$ & $8.84 \mu$ & $10.02 \mu$ & $11.79 \mu$ & $12.96 \mu$ & $14.14 \mu$ & $15.91 \mu$ & $17.68 \mu$ & $20.6 \mu$ & $22.5 \mu$ \\
\hline $15^{\circ}$ & 4.0 & 4.1 & 4.3 & 5.4 & 6.8 & 7.4 & 7.9 & 6.5 & 5.1 \\
$30^{\circ}$ & 7.9 & 9.1 & 10.8 & 11.9 & 12.9 & 13.5 & 13.5 & 8.1 & 5.4 \\
$45^{\circ}$ & 11.6 & 13.0 & 15.8 & 17.2 & 18.8 & 18.4 & 17.7 & 8.2 & 5.6 \\
$60^{\circ}$ & 15.0 & 17.2 & 20.3 & 21.7 & 23.0 & 22.1 & 19.6 & 8.2 & 5.6 \\
$70^{\circ}$ & 16.5 & 18.9 & 22.4 & 23.6 & 24.8 & 23.6 & 19.9 & 8.1 & 5.5 \\
$75^{\circ}$ & 16.0 & 18.4 & 22.2 & 23.5 & 25.0 & 23.5 & 19.6 & 8.0 & 5.4 \\
$80^{\circ}$ & 14.0 & 17.7 & 21.2 & 22.5 & 23.6 & 22.7 & 18.5 & 7.5 & 5.4 \\
$85^{\circ}$ & 8.2 & 10.7 & 14.6 & 16.5 & 18.1 & 18.9 & 15.5 & 5.4 & 5.2 \\
\hline \hline
\end{tabular}

It would seem impractical to narrow the slits narrower than the frequency interval $\Delta \nu^{\prime}$ corresponding to the theoretical resolving power of the spectrometer. For a prism spectrometer this is given by the Rayleigh formula.

$$
\Delta \nu^{\prime}=\frac{-c}{\lambda(\partial n / \partial \lambda) \tau} .
$$

If the absorption coefficient is high only the apex of the prism will transmit radiation and the value of $\tau$ to be substituted in the formula is much less than the actual value of $\tau$. In order to get an idea of how this limiting frequency interval, $\Delta \nu^{\prime}$, varies as one goes deeper in to the infrared it may be calculated using a value corresponding, for that wave-length in question, to a prism having a transmission of 95 percent. The values of $\tau_{T=.95}$ are given in Table IV

TABLE IV. Values of $\tau_{T=.95}$ and $\Delta \nu^{\prime}$ for various wave-lengths.

\begin{tabular}{|c|c|c|c|c|c|c|c|}
\hline$\lambda$ & $11.79 \mu$ & $12.96 \mu$ & $14.14 \mu$ & $15.91 \mu$ & $17.68 \mu$ & $20.6 \mu$ & $22.5 \mu$ \\
\hline $\begin{array}{l}\kappa \\
\tau_{T=95} \\
\Delta \nu^{\prime}\end{array}$ & $\begin{array}{c}0.004 \\
25 \\
.75\end{array}$ & $\begin{array}{l}0.0175 \\
5.72 \\
2.6\end{array}$ & $\begin{array}{l}0.0270 \\
3.71 \\
3.4\end{array}$ & $\begin{array}{l}0.065 \\
1.54 \\
6.2\end{array}$ & $\begin{array}{l}0.135 \\
.74 \\
9.9\end{array}$ & $\begin{array}{l}0.515 \\
.19 \\
26.4\end{array}$ & $\begin{array}{c}1.00 \mathrm{~cm}^{-1} \\
.10 \mathrm{~cm}^{-1} \\
38.4 \mathrm{~cm}^{-1}\end{array}$ \\
\hline
\end{tabular}

together with corresponding values of $\Delta \nu^{\prime}$. It is interesting to compare these limiting frequencies with the resolving power successively realized by Burmeister, Eva von Bahr, Brinsmade and Kemble, Imes and Randall, and Meyer and Bronk in their classical investigations of the fundamental oscillation band of $\mathrm{HCl}$. Their slit widths reduced to the same units used in Table IV are respectively $40,10,7,4$ and $3 \mathrm{~cm}^{-1}$.

When only the apex of the prism transmits appreciably and it is not possible to realize the limiting resolving power on account of the insensitivity of 
the receiver one may double or quadruple the energy passing through the exit slit by using two or four identical prisms side by side which are tall compared with $\tau$. Such an arrangement is shown in Fig. 1. Since it is much easier to make a plane parallel plate than to make a prism whose faces make a prescribed angle with each other, an alternative arrangement is illustrated by Fig. 2 . The back of each parallel plate is silvered by evaporation, the plates

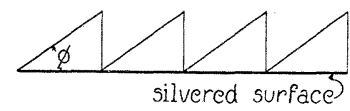

Fig. 1.

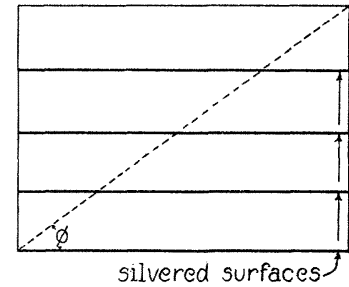

Fig. 2.

are waxed together and the ensemble cut and polished along the dotted line so as to give a lamellar prism with dispersing angle $35^{\circ}$ to $37.5^{\circ}$. This will form a Littrow half prism into which the light will not need to penetrate deeper than the thickness of the parallel plates used. It is, however, more advisable to use a prism which is more transparent in the desired spectral region.

\section{CONCLUSION}

From the values of $\epsilon$ given in Table III and the values of $\tau_{T=.95}$ and $\Delta \nu^{\prime}$ given in Table IV, we may draw the following conclusions.

1. A dispersing angle of $70^{\circ}$ to $75^{\circ}$ is best for a sylvine prism to be used from 8 to $22 \mu$. Table III shows the available energy for these prism angles to be greater for all wave-lengths out to $18 \mu$. Beyond $18 \mu$, there is so little difference in the available energy that the construction of a special $15^{\circ}$ prism for this region is not justified.

2. A sylvine prism with a $10 \mathrm{~cm}$ base is useful to $18 \mu$. In spite of the fact that the energy for illuminating the entrance slit of the spectrometer decreases with the fourth power of the wave-length, it will be observed from Table III that the energy available for activating a thermocouple increases. This means that the resolving power increases at longer wave-lengths as long as the available energy, rather than interference effects, places the limit on the resolving power of the spectrometer.

3. Values of $\tau_{T=.95}$ in Table IV show that only the apex of the prism transmits radiations. The available energy may be increased several fold by the use of a lamellar prism construction. This should make it possible to realize approximately the theoretical resolving power of one of the lamella. Such a lamellar prism would be particularly useful when the radiations are to be further dispersed with a grating. It is possible that the use of another prism material, such as potassium iodide (transparent to wave-lengths greater than $33 \mu$ ) would be better. However, since no material is yet known which is more transparent than potassium iodide it may be advisable to use a lamellar 
prism of this material in order to extend the region open to prism spectroscopy as far as possible. The optical properties of potassium bromide and potassium iodide are being investigated by the author so that a set of values of $\epsilon$ for all the infrared prism materials may be compiled.

4. An examination of the proportionality factor between $e$ and $\epsilon$ shows the available energy is inversely proportional to the focal length of the spectrometer collimator. This result indicates that a spectrometer of the Pfund type would give the best resolution. 